\title{
ON A GENERALIZED EGNELL INEQUALITY
}

\author{
ADAMARIA PERROTTA
}

Abstract. In this paper we prove an inequality which connects the $L^{p}$ norm of the gradient of a function $u$ with its $|x|^{v}$-weighted $L^{\frac{p(N+v)}{N-p}}$ norm and its $L^{p^{*}}$-weak norm. Here $1<p<N$, $-p<v \leqslant 0$ and $p^{*}=\frac{N p}{N-p}$. As a consequence we can provide an alternative proof of the Egnell inequality in $\mathbb{R}^{N}$.

Mathematics subject classification (2010): 49K20, 26D10, 39B62.

Keywords and phrases: Egnell inequality, one dimensional calculus of variations, isoperimetric inequality.

\section{REFERENCES}

[1] Adimurthi, N. Chaudhuri And M. Ramaswamy, An improved Hardy-Sobolev inequality and its application, Proc. Amer. Math. Soc., 130, (2002), 485-505.

[2] Adimurthi, S. FilipPas And A. Tertikas, On the best constant of Hardy-Sobolev inequalities, Nonlinear Anal., 70, (2009), 2826-2833.

[3] A. Alvino, Sulla diseguaglianza di Sobolev in spazi di Lorentz, Boll. Un. Mat. Ital. A, 14, 5 (1977), $148-156$.

[4] A. Alvino, On a Sobolev-type inequality, Atti Accad. Naz. Lincei Rend. Lincei Mat. Appl., 20, 4 (2009), 379-386.

[5] A. Alvino, R. Volpicelli And B. Volzone, On Hardy inequalities with a remainder term, Ric. Mat., 59, (2010), 265-280.

[6] T. Aubin, Problème isopérimétriques et espaces de Sobolev, J. Diff. Eq., 11, (1976), 573-598.

[7] G. Barbatis, S. FilipPas AND A. Tertikas, A unified approach to improved $L^{p}$ Hardy inequalities with best constant, Trans. Amer. Math. Soc., 356, 6 (2004), 2169-2196.

[8] G. A. BLISs, An integral inequality, J. London Math. Soc., 5, (1930), 40-46.

[9] H. BREZIS AND E.H. LIEB, Sobolev inequalities with remainder terms, J. Funct. Analysis, 62, (1985), $73-86$.

[10] H. Brezis And L. Niremberg, Positive solutions of nonlinear equations involving critical Sobolev exponents, Comm. Pure Appl. Math., 36, (1983), 437-477.

[11] H. BReZIS AND J.L. VAZQUeZ, Blow-up solutions of some nonlinear elliptic problems, Rev. Mat. Univ. Complut. Madrid, 2, (10) (1997), 443-469.

[12] X. CABRÉ AND Y. MARTEL, Weak eigenfunctions for the linearization of extremal elliptic problems, J. Funct. Anal., 156, (1998), 30-56.

[13] L. Caffarelli, R. Kohn And L. Nirenberg, First order interpolation inequality with weights, Compositio Math., 53, (1984), 259-275.

[14] F. CATRINA AND Z. Q. WANG, On the Caffarelli-Kohn-Niremberg inequalities: sharp constants, existence (and nonexistence), and symmetry of extremal functions, Comm. Pure Appl. Math., 54, (2001), 229-258.

[15] N. Chaudhuri AND M. RAmASWAmy, Existence of positive solutions of some semilinear elliptic equations with singular coefficients, Proc. Roy. Soc. Edinburgh Sect. A 131, 6, (2001), 1275-1295.

[16] K. M. ChONG AND N. M. Rice, Equimeasurable rearrangements of functions, Queen's Papers in Pure and Applied Mathematics, 28, Queen's University, Kingston, Ontario, 1971.

[17] S. Cuomo And A. Perrotta, On best constant in Hardy inequalities with a remainder term, Nonlinear Anal., 74, (2011), 5784-5792. 
[18] H. EGNELL, Elliptic boundary value problems with singular coefficients and critical nonlinearities, Indiana Univ. Math. J., 38, (1989), 235-251.

[19] H. Egnell, F. Pacella And M. Tricarico, Some remarks on Sobolev Inequalities, Nonlinear Anal., 13, (1989), 671-681.

[20] S. Filippas, V. G. MaZ'Ja And A. Tertikas, Sharp Hardy-Sobolev inequalities, C. R. Math. Acad. Sci. Paris, 339, (2004), 483-486.

[21] S. Filippas And A. Tertikas, Optimizing improved Hardy inequalities, J. Funct. Anal., 192, (2002), 186-233, J. Funct. Anal., 255, (2008), 2095 (Corrigendum).

[22] F. Gazzola, H. C. Granau And E. Mitidieri, Hardy inequalities with optimal constants and remainder terms, Trans. Amer. Math. Soc., 356, 6 (2004), 2149-2168.

[23] N. Ghoussoub ANd A. Moradifam, On the best possible remaining term in the Hardy inequality, Proc. Natl. Acad. Sci. USA, 105, (2008), 13746-13751.

[24] B. Kawhol, Rearrangements and convexity of level sets in P.D.E., Lecture Notes in Mathematics, 1150, Springer, Berlin, 1985.

[25] G. H. HARDY, Notes on some points in the integral calculus, Messenger Math., 48, (1919), 107-112.

[26] G. H. Hardy, J. E. Littlewood And G. Polya, Inequalities, Cambridge University Press, Cambridge, 1964.

[27] T. HoriUchI, Best constant in weighted Sobolev inequality, Proc. Japan Acad. Ser. A Math. Sci., 72, (1996), 208-211.

[28] V. G. MaZ'Ja, Sobolev Spaces, Springer-Verlag, Berlin, 1986.

[29] A. PERROTtA AND B. VOlzone, A note on a Sobolev Inequality with a remainder term for functions vanishing on part of the boundary, Rend. Acc. Sci. fis. Mat. Napoli, LXXIV, (2007), 35-50.

[30] V. Radules Ku, D. SMets AND M. Willem, Hardy-Sobolev inequalities with a remainder term, Topol. Methods Nonlinear Anal., 20, 1 (2002), 145-149.

[31] H. SAGAN, Introduction to the Calculus of Variations, Dover Pubblications, Inc., New York, 1969.

[32] G. Talenti, Best constant in Sobolev inequality, Ann. Mat. Pura Appl., 110, 4 (1976), 353-372.

[33] J.L. VAZQueZ, Domain of existence and blow-up for the exponential reaction diffusion equation, Indiana Univ. Math. J., 48, (1999), 677-709.

[34] J.L. VAzQueZ And E. ZuAzuA, The Hardy inequality and the asymptotic behaviour of the heat equation with an inverse square potential, J. Funct. Anal., 173 (2000), 103-153. 